\title{
Single I mage Dehazing Using Dark Channel Prior and Minimal Atmospheric Veil
}

\author{
Xiao Zhou, Chengyou Wang*, Liping Wang, Nan Wang, and Qiming Fu \\ School of Mechanical, Electrical and Information Engineering, Shandong University, Weihai 264209, China \\ [e-mail: zhouxiao@sdu.edu.cn,wangchengyou@sdu.edu.cn, sduwlp@163.com,641568764@qq.com, \\ fqmsdu@163.com] \\ *Corresponding author: Chengyou Wang
}

Received July 5, 2015; revised October 10, 2015; revised October 17, 2015; revised October 21, 2015; accepted October 27, 2015; published January 31, 2016

\begin{abstract}
Haze or fog is a common natural phenomenon. In foggy weather, the captured pictures are difficult to be applied to computer vision system, such as road traffic detection, target tracking, etc. Therefore, the image dehazing technique has become a hotspot in the field of image processing. This paper presents an overview of the existing achievements on the image dehazing technique. The intent of this paper is not to review all the relevant works that have appeared in the literature, but rather to focus on two main works, that is, image dehazing scheme based on atmospheric veil and image dehazing scheme based on dark channel prior. After the overview and a comparative study, we propose an improved image dehazing method, which is based on two image dehazing schemes mentioned above. Our image dehazing method can obtain the fog-free images by proposing a more desirable atmospheric veil and estimating atmospheric light more accurately. In addition, we adjust the transmission of the sky regions and conduct tone mapping for the obtained images. Compared with other state of the art algorithms, experiment results show that images recovered by our algorithm are clearer and more natural, especially at distant scene and places where scene depth jumps abruptly.
\end{abstract}

Keywords: Image dehazing, dark channel prior, atmospheric veil, guided filtering, bilateral filtering, tone mapping

This work was supported by the National Natural Science Foundation of China (Grant No. 61201371), the Natural Science Foundation of Shandong Province, China (Grant No. ZR2015PF004), the promotive research fund for excellent young and middle-aged scientists of Shandong Province, China (Grant No. BS2013DX022), the Fundamental Research Funds of Shandong University (Grant No. 2014ZQXM008), and the Development Program for Outstanding Young Teachers in School of Mechanical, Electrical and Information Engineering, Shandong University, Weihai. 


\section{Introduction}

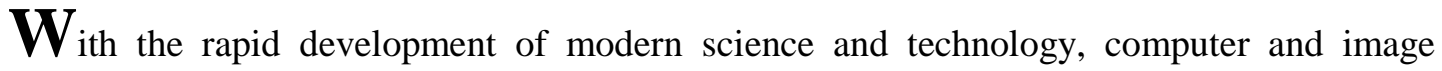
processing technology are widely used in science, military and other fields. As the digital equipments become cheaper and cheaper, the application of computer vision also plays an increasingly important role in people's life. Haze or fog is a common natural phenomenon formed by the increased size of fine suspended particles in the atmospheric media which affect atmospheric absorption, emission and scattering of light. On the one hand, outdoor images acquired with the presence of these scenarios are often of low quality, such as low image resolution and color distortion. The outdoor imaging equipment is generally distant from the observation object. And this makes outdoor imaging equipment more vulnerable to the impact of bad weather. The presence of fog or haze will lead to substantially reduce of the visibility of the image scene, which will become a major problem. On the other hand, the existing visual application system is built on the basis of clear and high quality images. So high quality images are needed. Therefore, how to solve the bad effect resulted from the bad weather is the main problem in many computer vision applications, such as video surveillance, remote sensing, navigation, target identification, etc. In addition, even in the sunny weather conditions, due to the presence of dust and water vapor in the air, as well as the hardware system noise, the images acquired by imaging device are degraded and affect normal work of the visual application. Updating the existing hardware equipment which can operate in adverse weather conditions needs large required work and high cost. So it is not realistic. Thus, haze removal method to the degraded image has become feasible at present. Although many proposed image dehazing methods have achieved good results, these methods still have some shortcomings in terms of reconstructed image quality and computing speed. Therefore, image dehazing is an urgent problem to be solved.

Image dehazing methods include two categories on the basis of image enhancement and image restoration. The method based on image enhancement improves the quality of image mainly by image contrast enhancement or color enhancement, and has developed many mature algorithms, such as histogram equalization algorithm [1] and multi-scale color image enhancement algorithm [2]. But this method does not consider image degradation factors, and may lead to loss of original part information, and the result of dehazing is distorted. So at present, the method based on image restoration is widely used, which establishes the atmospheric scattering model [3] and inverse image degradation process to improve the visual effect of foggy images. The main problem of this method is that the atmospheric scattering model is an underdetermined equation, and other auxiliary information is needed to recover the foggy images. To solve this problem, many methods have been proposed: (1) the use of multiple foggy images. This method mainly has two implementations. One is to install the rotate polarization filter in front of the imaging equipment [4], which can obtain the fog degradation parameters according to the two images (or more than two) with different degree of polarization under the same scene. Another is to obtain multiple frame images of the same scene in different media [5] and extract more constraint conditions from the image color model and the structural characteristics of the multiple frame images in order to recover the foggy images; (2) the use of the known prior information to dehaze. This method directly provides depth information of the captured scene by establishing the 3D space model [6] of the captured scene. But these two kinds of methods mentioned above are not suitable for dynamic scenes dehazing. Multiple images dehazing needs to wait for calculating the maximum and minimum 
polarization angle or the emergence of different media. The constructed 3D model based on prior information method will fail due to the scene changing. While for single image, it achieves good performance. Therefore, many researchers focus on the field of single image dehazing.

To improve the efficiency of single image dehazing, several methods have been proposed. Through statistics, Tan [7] found that clear images had higher contrast compared with foggy images, thus he maximized the local contrast of the recovered image for enhancing image visibility. However, the algorithm does not recover the image from the physical significance. So the disadvantage is that the color of the restored image is often oversaturated and partial information may lose. Based on the assumption that the propagation of light and shading parts of the target surface are locally uncorrelated, Fattal [8] used independent component analysis (ICA) to estimate the medium transmission and then recovered the foggy images. As this method required sufficient color information, it could not process gray level images. In addition, the proportion of surface reflection light is smaller than the ambient light in the dense fog, so the method is not suitable for the dense fog image to dehaze. He et al. [9] proposed a single image haze removal method based on dark channel prior and obtained impressive results. However, this method needs to refine the transmission map through soft matting technique [10], which could be computational intensive. To solve this problem, He et al. [11] proposed a guided filter, and found that the output of a guided filter could be an approximate solution of the Laplacian matting optimization equation. Thus, refining the transmission map using guided filter greatly reduces the time complexity. However, since the original foggy image is chosen as the guidance image, the texture details of the recovered image are weakening and the haze removal results may not be the best. Tarel and Hautiere [12] proposed a fast dehazing algorithm based on atmospheric veil. This algorithm recovered the foggy images through estimating atmospheric veil from minimum color component using median filter. This algorithm is very efficient, but since the median filter is not conformal and edge-preserving, the estimating atmospheric veil does not reflect the depth information of the scene accurately. As a result, in some small edge regions with large depth jumps, the desirable dehazing results cannot be achieved. The sky region processed by these algorithms tends to be degraded by serious noise and color distortion. Based on the in-depth analysis on the degradation, Wang et al. [13] proposed an improved dehazing algorithm which first segmented the haze image into the sky and non-sky region, then estimated the transmission coefficients of the two regions separately, and last combined of the regions with a refining step. Li et al. [14] proposed a weighted guided image filter (WGIF) for the problem that amplification of noise in sky regions is addressed for single image haze removal. Li and Zheng [15] also proposed a single image haze removal algorithm by introducing an edge-preserving decomposition technique to estimate the transmission map for a haze image. Zhu et al. [16] proposed a simple but powerful color attenuation prior for haze removal from a single input hazy image. By creating a linear model to model the scene depth of the hazy image under this novel prior method and learning the parameters of the model with a supervised learning method, the depth information can be well recovered.

The scene depth information of the degraded image is an important clue to haze removal. In this paper, we propose an improved single image haze removal algorithm through reconstructing a more accurate atmospheric veil. Since the atmospheric veil mainly depends on the scene depth information of original foggy image, which tends to be constant most of time, the desirable atmospheric veil should be smooth except along edges with large depth jumps. Based on this assumption, our basic idea is to obtain a more accurate atmospheric veil from minimum color component and dark channel by using some filter tools. The minimum 
color component map of an original foggy image contains the edge information of the foggy image, and minimum color component map filtered by bilateral filter will reflect the the depth information of the scene more accurately than minimum color component map itself; the dark channel of an original foggy image can be considered to be a rough estimation of the atmospheric veil; in guided filter, we regard the former as the guidance image, and the latter as input image, the filtering output is the final atmospheric veil we need. As we reconstruct a more accurate atmospheric veil, better dehazing results will be obtained. What's more, in our dehazing method, we also use quad-tree subdivision method to estimate the atmospheric light more accurately.

The rest of this paper is organized as follows. Section 2 introduces the background of image dehazing and the image dehazing physical model. Section 3 starts with brief review of several fog removal schemes and atmospheric light value estimation methods, and then analyzes their advantages and disadvantages. In Section 4, we propose an improved image dehazing method based on atmospheric veil and dark channel prior. Experimental results of the proposed method are presented in Section 5. Couclusions and remarks on possible further work are given finally in Section 6.

\section{Background of Image Dehazing}

A single image dehazing is based on atmospheric scattering model, through estimation of scene information to restore the degraded image. The atmospheric attenuation model describes the way light gets attenuated as it traverses from a scene point to the observer.

The light received by human eyes and imaging equipment is mainly composed of two parts. It is defined by

$$
I_{c}(x)=A_{c} \rho(x) \mathrm{e}^{-\beta d(x)}+A_{c}\left[1-\mathrm{e}^{-\beta d(x)}\right],
$$

where $x$ indicates any pixel point within the scene. $c \in\{r, g, b\}$ is color channel index. $I_{c}(x)$ is the observed light intensity. $A_{c}$ is the global atmospheric light, and $\rho(x)$ is a function that describes the reflectance properties. $\beta$ is called the sacttering coefficient of the amosphere. And $d(x)$ is the depth from the scene point to the observer, called scene depth. The first part is attenuated irradiance model [3]. Due to atmospheric scattering, a fraction of light flux is removed from the incident beam. The unscattered flux, called direct transmission, is transmitted to the observer. The attenuated irradiance is illustrated by the solid arrow from the scene to the sensor in Fig. 1. The second atmospheric scattering model is called the airlight model. The airlight model quantifies how a column of atmosphere acts as a light source by reflecting environmental illumination towards an observer. Referring to the dotted arrows in Fig. 1, the light reflected into the line of sight is integrated along the entire path length $d$, from the scene to the observer.

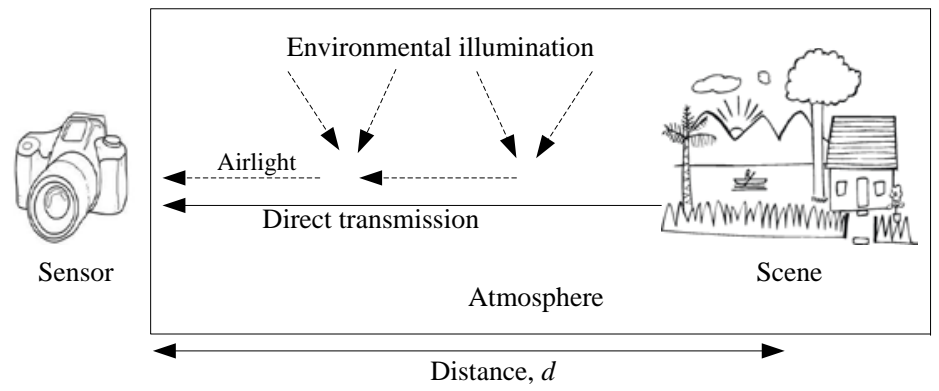

Fig. 1. Scattering of light by atmospheric particles. 
Incident light attenuation and too much environmental light involved in imaging are the main reasons why foggy images are degraded. Narasimhan and Nayar [3] first applied atmospheric imaging model to image dehaze, and gave the atmospheric scattering physical model under fog or haze weather conditions,

$$
I_{c}(x)=J_{c}(x) t(x)+A_{c}[1-t(x)]
$$

where $I_{c}(x)$ is the observed foggy image. $J_{c}(x)=A_{c} \rho(x)$ is the scene radiance, and $t(x)=\mathrm{e}^{-\beta d(x)}$ is the medium transmission describing the portion of the light that is not scattered and reaches the camera. It also includes two parts: the direct attention term and the atmospheric veil term. The term $J_{c}(x) t(x)$ is the direct attention, which indicates that haze will induce the scene radiance to attenuate exponentially with the scene depth $d(x)$ in the medium. Its performance is multiplicative effect. The second term $A_{c}[1-t(x)]$ is the atmospheric veil term, which causes fuzzy, color shift, and distortion in the scene. Its performance is additive effect. It indicates that environmental light participates in imaging, but does not show the scene feature.

\section{Previous Fog Removal Scheme Review}

\subsection{Image Dehazing Scheme Based on Atmospheric Veil}

The goal of haze removal is to recover $J_{c}(x)$ from observed foggy image $I_{c}(x)$. Tan [7] pointed out that if depth information is not available, it is difficult to calculate atmospheric scattering coefficient $\beta$ and the depth of the scene $d(x)$, so the atmospheric veil was introduced, as shown in Eq. (3)

$$
V_{c}(x)=A_{c}[1-t(x)]
$$

Then the atmospheric scattering model of the fog imaging can be rewritten as

$$
I_{c}(x)=J_{c}(x)\left[1-\frac{V_{c}(x)}{A}\right]+V_{c}(x)
$$

A desired atmospheric veil $V_{c}(x)$ should meet the following two constraints: (1) the value of $V_{c}(x)$ is positive at each pixel; (2) the value of $V_{c}(x)$ is not higher than the minimum component of $I_{c}(x), W(x)$. It can be shown by:

$$
\begin{gathered}
0 \leq V_{c}(x) \leq W(x), \\
W(x)=\min _{c \in\{r, g, b\}} I_{c}(x) .
\end{gathered}
$$

By Eq. (6), the minimum color component is the minimum value of RGB pixel value for each pixel in the foggy image. Minimum color component can reflect the scene depth information well and correspond roughly to the atmospheric veil $V_{c}(x)$, so it can be used to estimate the intensity of atmospheric veil $V_{c}(x)$. According to global atmospheric light $A_{c}$ 
and atmospheric veil $V_{c}(x)$, the fog-free image can be recovered by:

$$
J_{c}(x)=A_{c} \frac{I_{c}(x)-V_{c}(x)}{A_{c}-V_{c}(x)} .
$$

Tan [7] recovered the the foggy image according to its minimum color component, by maximizing the local contrast of the restored image. Because he did not recover foggy image physically, it easily leads to the resulting image oversaturated and distorted. However, the concept of minimum color component, as well as the idea of atmospheric veil, is still used by many researchers for image dehazing, and good results have been achieved. Based on this, this paper summarizes and analyzes some dehazing methods, and discusses the effect of the dehazing.

\subsubsection{Tarel's Method to Image Dehaze}

In most cases, the atmospheric veil is smooth, and the atmospheric veil has a large jump only when the depth value changes abruptly. In Tarel's image dehazing method [12], atmospheric veil $V_{c}(x)$ is inferred based on minimum color component using median filter. Median filter is a common low pass filter and it is greatly similar to the mean filter, so Tarel used median filter to yield desirable $V_{c}(x)$ instead of mean filter. The specific process to obtain atmospheric veil is summarized as follows:

$$
\begin{gathered}
A(x)=\operatorname{med}_{s_{v}} W(x), \\
B(x)=A(x)-\operatorname{med}_{s_{v}}[W(x)-A(x) \mid], \\
V_{c}(x)=\max \{\min [p B(x), W(x)], 0\},
\end{gathered}
$$

where $s_{v}$ is the size of the square or disc window used in the median filter, and $W(x)$ is the minimum color component map value at pixel $x$. From Eq. (8), we know that the local average of $W$ is $A(x)$; Eq. (9) indicates that atmospheric veil is approximate to the difference of the local average and the standard deviation of $W$; Eq. (10) is obtained due to the constraint $0 \leq p B(x) \leq W(x)$, where a scale factor $p \in[0,1]$ is introduced to control the strength of visibility restoration. The $p$ is usually fixed to 0.90 .

Fig. 2 shows the dehazed images based on atmospheric veil using differet methods. Fig. 2(c) shows the the atmospheric veil obtained through Tarel's Method. After median filtering, texture details are removal and obtained atmospheric veil is generally smooth. With atmospheric veil estimated, the recovered fog-free image is showed in Fig. 2(d). We can find that dehazed image is clear and natural except at the gaps among the leaves. There are still some fogs in the gap among the leaves. The reason is that as median filter itself is not conformal, lots of edge information, where the scene depth value changes abruptly, in the atmospheric veil $V_{c}(x)$ are weakened after twice median filtering, especially at the edges among the leaves. If the edge information is missing, haze cannot be removed completely among the edge regions. In summary, the atmospheric veil is the key to recover foggy images. The main advantage of Tarel's image dehazing method compared with others is its speed, which allows real time processing. 


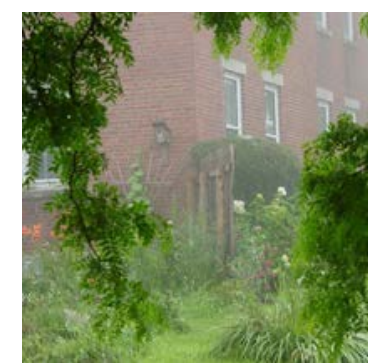

(a)

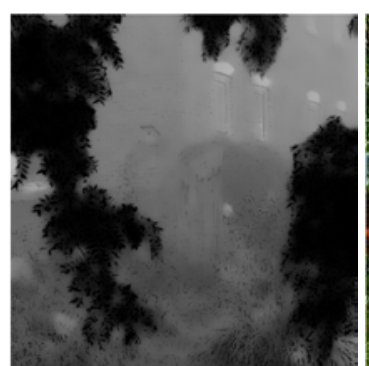

(c)

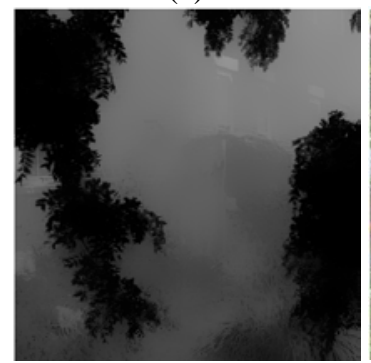

(g)

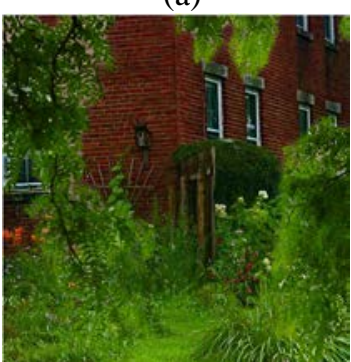

(d)

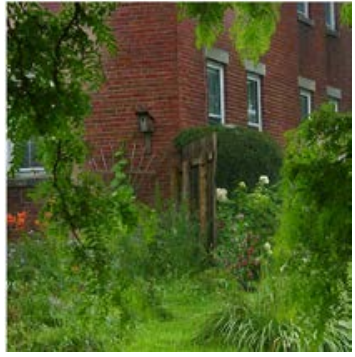

(h)

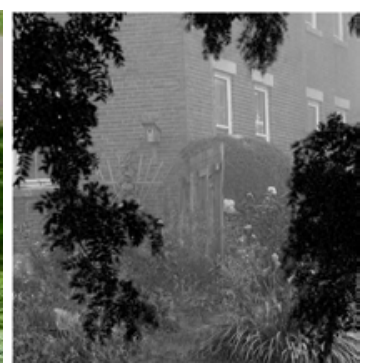

(b)

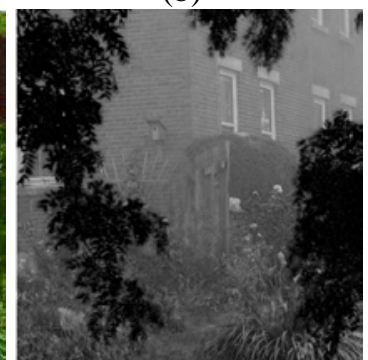

(e)

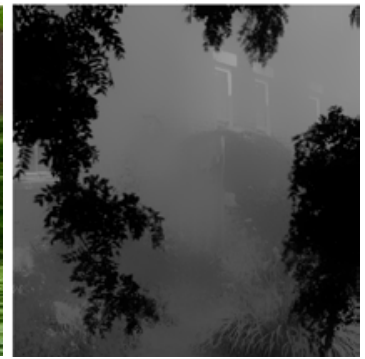

(i)

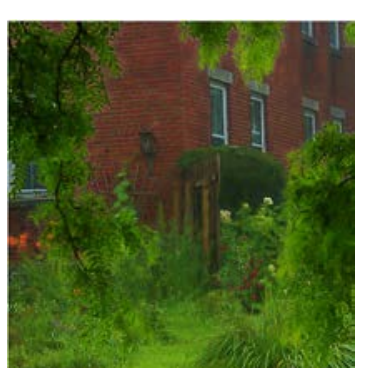

(f)

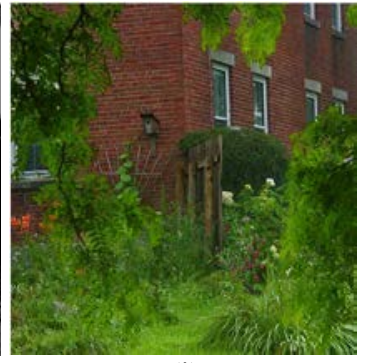

(j)

Fig. 2. Image dehazing based on atmospheric veil: (a) is the input foggy image; (b) is the minmum color component of input image; (c), (e), (g), and (i) are the atmospheric veil obtained through Tarel's method, Sun's method, Xiao's method based on joint bilateral filtering, Xiao's method based on guided joint bilateral filtering, respectively; (d), (f), (h), and (j) are the dehazed images obtained by Tarel's method, Sun's method, Xiao's method based on joint bilateral filtering, and Xiao's method based on guided joint bilateral filtering, respectively.

\subsubsection{Sun's Method to Image Dehaze}

It can be inferred that the variation of the atmospheric veil mainly depends on the scene depth. Thus, the desired atmospheric veil should be smooth and its intensity should gradually vary according to the scene depth. As shown in Fig. 2(b), there is much texture information as well as the edge information remaining in the $W(x)$. The edge information is important as they suppose possible large depth jumps among the objects, such as the edges between the leaves and the wall in Fig. 2. Thus, when we infer the $V_{c}(x)$ from the $W(x)$, it is necessary to protect edge information and remove texture information in $W(x)$. In Tarel's method, most texture details in the estimated atmospheric veil are removal after median filtering, including some important edge information.

In order to keep edge information in estimated atmospheric veil, Sun et al. [17] used the bilateral filter instead of median filter to obtain atmospheric veil. In Sun's method, the process to obtain atmospheric veil is identical with Tarel's method except filtering process. The bilateral filter, a nonlinear filter, originally introduced by Tomasi and Manduchi [18], has been 
widely used in computer graphics and computer vision community due to its characteristic of smoothing the image while preserving edges. The obtained atmospheric veil by Sun's method is shown in Fig. 2(e). Since the bilateral filter can keep most detail information, edges are preserved well.

The dehazed images obtained through Sun's method is shown in Fig. 2(f). It can be seen that, bilateral filtering can keep the existing jumps well, and the outline of the tiny leaves is also effectively restored. The fog in the gap among the leaves are removed completely. However, compared with the median filtering, it can be found that the strength of smoothing by bilateral filtering is not enough. A lot of texture details information, such as brick texture information on the wall, is preserved in the atmospheric veil, which leads to the texture of the recovered fog-free image being filtered out. As Fig. 2(f) shows, after applying the Sun's method, the cracks among the bricks on the wall become unclear due to too much corresponding texture details preserved in atmospheric veil after bilateral filtering.

\subsubsection{Xiao's Method to Image Dehaze}

Xiao and Gan [19] think one appropriate filtering method should keep the atmospheric veil smooth, preserve the depth jumps, and remove the redundant texture information. To reach the above goals, they try to correct the atmospheric veil $V_{c}(x)$. The purpose of this correction operator is to restore the edge information in $V_{c}(x)$, and reduce texture details in $V_{c}(x)$, as texture information does not suggest depth variation. They first use a bilateral filter on $W(x)$ to filter out some texture details, while the edge features can be well preserved. Then, they take the minimum color component $W(x)$ filtered by bialteral filter as the guidance image, which contains the edge information of the source image, and propose a "guided joint bilateral filter" to enhance the atmospheric veil $V_{c}(x)$ achieved by Eq. (10). They try to add edge information of the original image to $V_{c}(x)$, meanwhile reducing texture details of $V_{c}(x)$.

In Xiao's method based on guided joint bilateral filter, the input image is the atmospheric veil $V_{c}(x)$ obtained by Tarel's method and the guidance image is the minimum color component filtered by bialteral filter. After guided joint bilateral filtering, $V_{c}(x)$ is further refined, which is shown in Fig. 2(i). In [19], with the same guidance image and input image, joint bilateral filter, as a comparison, is also used to refine the atmospheric veil $V_{c}(x)$ achieved by Tarel's method. The atmospheric veil refined by joint bilateral filter is shown in Fig. 2(g), and corresponding dehazed image is shown in Fig. 2(h). Compared with joint bilateral filter, "guided joint bilateral filter" acquires better results to recovery edge information from atmospheric veil $V_{c}(x)$. With a more accurate atmospheric veil obtained, from Fig. 2(j), it can be seen that Xiao's method based on guided joint bilateral filter has the best result in image dehazing under current discussion. The fog in gaps between leaves is completely removed and the texture details of the recovered scene are clear.

\subsection{Image Dehazing Scheme Based on Dark Channel Prior}

Through observation and mathematical statistics conduction on a large number of outdoor haze-free images, He et al. [9] discovered that: in most of the non-sky patches, at least one color channel has some pixels whose intensity are very low and close to zero. Equivalently, the minimum intensity in such a patch is close to zero. They first gave the definition of dark channel. For an arbitrary image $\boldsymbol{J}$, its dark channel $J_{\text {dark }}(x)$ is given by: 


$$
J_{\text {dark }}(x)=\min _{y \in \Omega(x)}\left[\min _{c \in\{r, g, b\}} J_{c}(y)\right],
$$

where $J_{c}(x)$ is a color channel of $\boldsymbol{J}$ and $\Omega(x)$ is a local patch centered at $x$. A dark channel is the outcome of two minimum operators: $\min _{c \in\{r, g, b\}}$ is performed on each pixel and $\min _{y \in \Omega(x)}$ is a minimum filter. The minimum operators are commutative. Using the concept of a dark channel, their observation says that if $\boldsymbol{J}$ is an outdoor haze-free image, except for the sky region, the intensity of $J$ 's dark channel is low and tends to be zero: $J_{\text {dark }}(x) \rightarrow 0$.

We assume that the atmospheric light $A_{c}$ is given. Several methods to estimate $A_{c}$ will be introduced in Section 3.3. In [9], they first normalize the haze imaging Eq. (2) by $A_{c}$. They further assume that the medium transmission in a local patch $\Omega(x)$ is constant. Then, they calculate the dark channel on both sides. Equivalently, they put the minimum operators on both sides:

$$
\min _{y \in \Omega(x)}\left\{\min _{c \in\{r, g, b\}}\left[\frac{I_{c}(y)}{A_{c}}\right]\right\}=t(x) \min _{y \in \Omega(x)}\left\{\min _{c \in\{r, g, b\}}\left[\frac{J_{c}(y)}{A_{c}}\right]\right\}+1-t(x),
$$

where $A_{c}$ is a color channel, $J_{c}$ is haze-free image, and the dark channel of $J_{c}$ is close to zero due to the dark channel prior,

$$
\min _{y \in \Omega(x)}\left\{\min _{c \in\{r, g, b\}}\left[\frac{J_{c}(y)}{A_{c}}\right]\right\} \rightarrow 0
$$

Last, they estimate the medium transmission $t(x)$ simply by

$$
t(x)=1-\min _{y \in \Omega(x)}\left\{\min _{c \in\{r, g, b\}}\left[\frac{I_{c}(y)}{A_{c}}\right]\right\} .
$$

In practice, even on clear days the atmosphere is not absolutely free of any particle. So the haze still exists when we look at distant objects. Moreover, the presence of haze is a fundamental cue for human to perceive depth. This phenomenon is called aerial perspective. If the haze is removed thoroughly, the image may seem unnatural and we may lose the feeling of depth. So in [9], a constant parameter $\omega(0<\omega<1)$ is introduced into Eq. (14) to optionally keep a very small amount of haze for the distant objects, and the final estimation of the medium transmission is given by

$$
t(x)=1-\omega \min _{y \in \Omega(x)}\left\{\min _{c \in\{r, g, b\}}\left[\frac{I_{c}(y)}{A_{c}}\right]\right\} .
$$

In [9], the $\omega$ is fixed to 0.95 . And the the fog-free image can be recovered by

$$
J_{c}(x)=\frac{I_{c}(x)-A_{c}}{t(x)}+A_{c} .
$$


In this image dehazing scheme, in order to refine transmission map obtained by the the dark channel efficiently, the guided filter [11] is adopted, where transmission map calculated from the dark channel is the input image and the foggy image is the gudiance image. Fig. 3 shows the experimental results of He's dehazing method. In order to compare He's work with the image dehazing method mentioned above expediently, what we give in Fig. 3 is the atmospheric veil which is calculated from given transmission map by Eq. (3). As Fig. 3(d) shows, the defogging result of the He's method is good, texture details in recovered image are obvious and most fogs have been removed. However, we can see that compared with the atmospheric veil in Fig. 2(i), the atmospheric veil in Fig. 3(c) has less edges and more textures. Therefore, Xiao's method based on guided joint bilateral filter obtains better results in image dehazing.

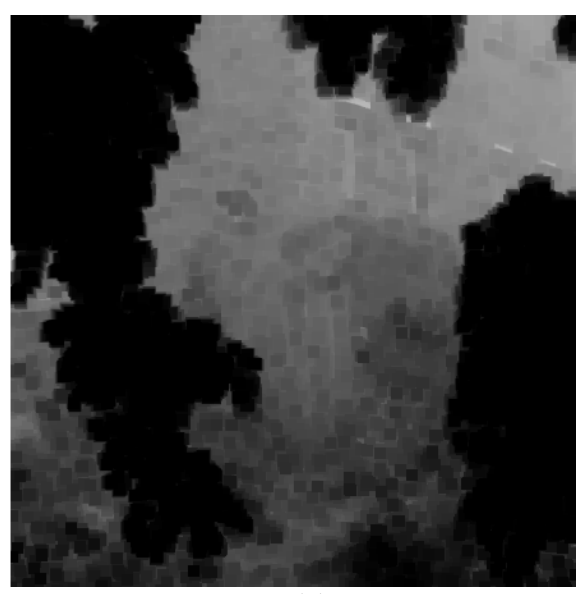

(a)

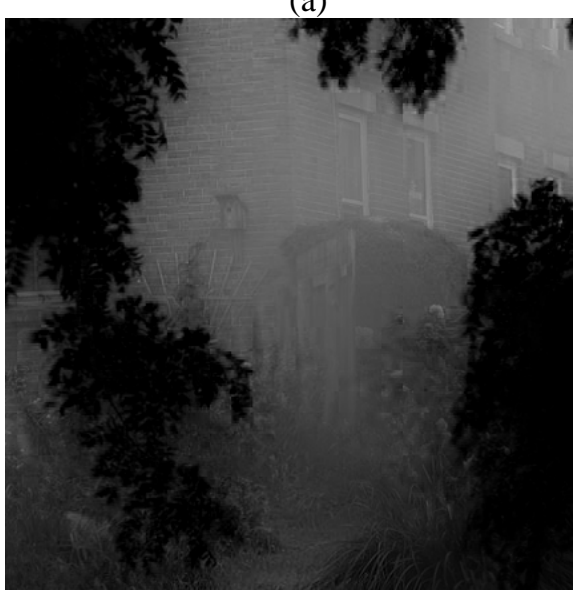

(c)

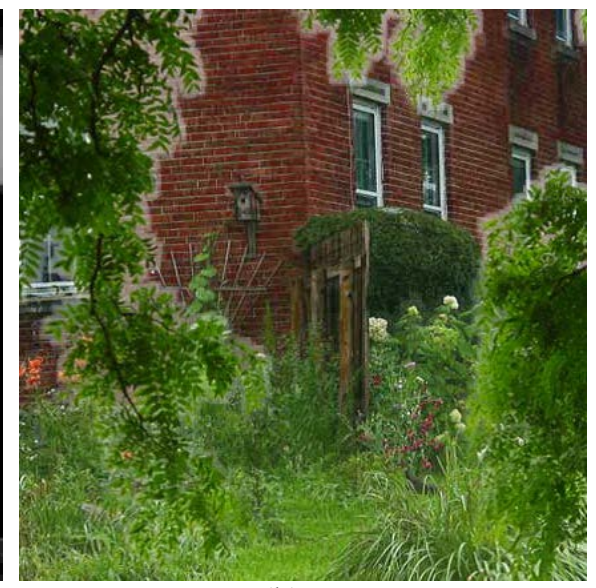

(b)

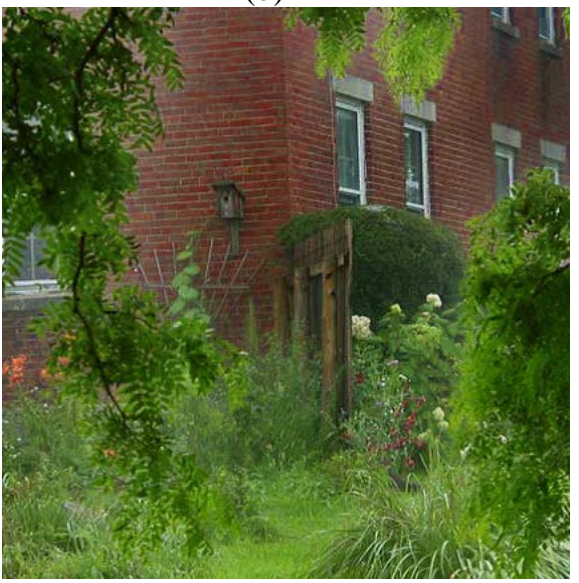

(d)

Fig. 3. Image dehazing based on dark channel prior: (a) and (c) are the dark channel (the roughly estimated atmospheric veil equivalently), and the atmospheric veil calculated form refined transmission map, respectively; (b) and (d) are the dehazed images obtained by rough transmission map (calculated directly by the dark channel), and refined transmission map, respectively. 


\subsection{The Atmospheric Light Estimation}

In previous discussion, we have assumed that the atmospheric light $A_{c}$ is known. Here, the method to estimate atmospheric light $A_{c}$ will be discussed. In many single image dehazing methods, the overall atmospheric light $A_{c}$ is usually estimated from pixels with most dense haze. In Tan's work [7], considering that in many situations of bad weather, particularly in daylight where the sky is usually overcast, Tan ignored the presence of the sunlight, and assumed that the atmospheric light $A_{c}$ is globally constant. According to Eq. (2), this global value of $A_{c}$ can be obtained from pixels that have the highest intensity in the input image. But in practice, the brightest pixels in an image could be on a white car or a white building. In this situation, the brightest pixels of the whole image can be brighter than the atmospheric light. He et al. [9] proposed that we can improve the atmospheric light estimation accuracy by using the dark channel of hazy image. He used following steps to determine the atmospheric light $A_{c}$ :

(1) First, pick the top $0.1 \%$ brightest pixels in the dark channel and these pixels are most haze-opaque;

(2) Among these pixels, the pixel with highest intensity in the input image is selected as the atmospheric light $A_{c}$.

He's method based on the dark channel prior is more robust than the "brightest pixel" method. However, He's algorithm is vulnerable to interference of white objects and artificial light sources in the image. Therefore, inspired by the work in [20], this paper estimates the atmospheric light using the quad-tree subdivision method. The specific process of using the quad-tree subdivision method for estimating the global atmospheric light intensity is summarized as follows:

Step 1. Divide the input foggy image into four equal rectangular regions (sub-blocks);

Step 2. Calculate the average pixel values of each sub-block, and the highest value is denoted as $A_{\max }$. Meanwhile, retain the the sub-block with the highest value $A_{\max }$;

Step 3. If $A_{\max }$ is smaller than the preset threshold and the size of sub-block retained in the Step 2 is not less than the preset minimum window size, the retained sub-block will go back to the Step 1 and be further divided. Otherwise, continue to Step 4;

Step 4. Convert the input image from RGB into YCbCr format, and in the region corresponding to the final retained sub-block, we select the maximum value in the luminance channel as global atmospheric light $A_{c}$.

In Fig. 4, the red rectangles indicate where these different methods automatically obtain the atmospheric light. From Fig. 4, we can see that the quad-tree subdivision method obtains better results and manages to detect the most haze-opaque regions which can provide a good approximation of $A_{c}$. The obtained global atmospheric light $A_{c}$ values with different methods are shown in Table 1. 

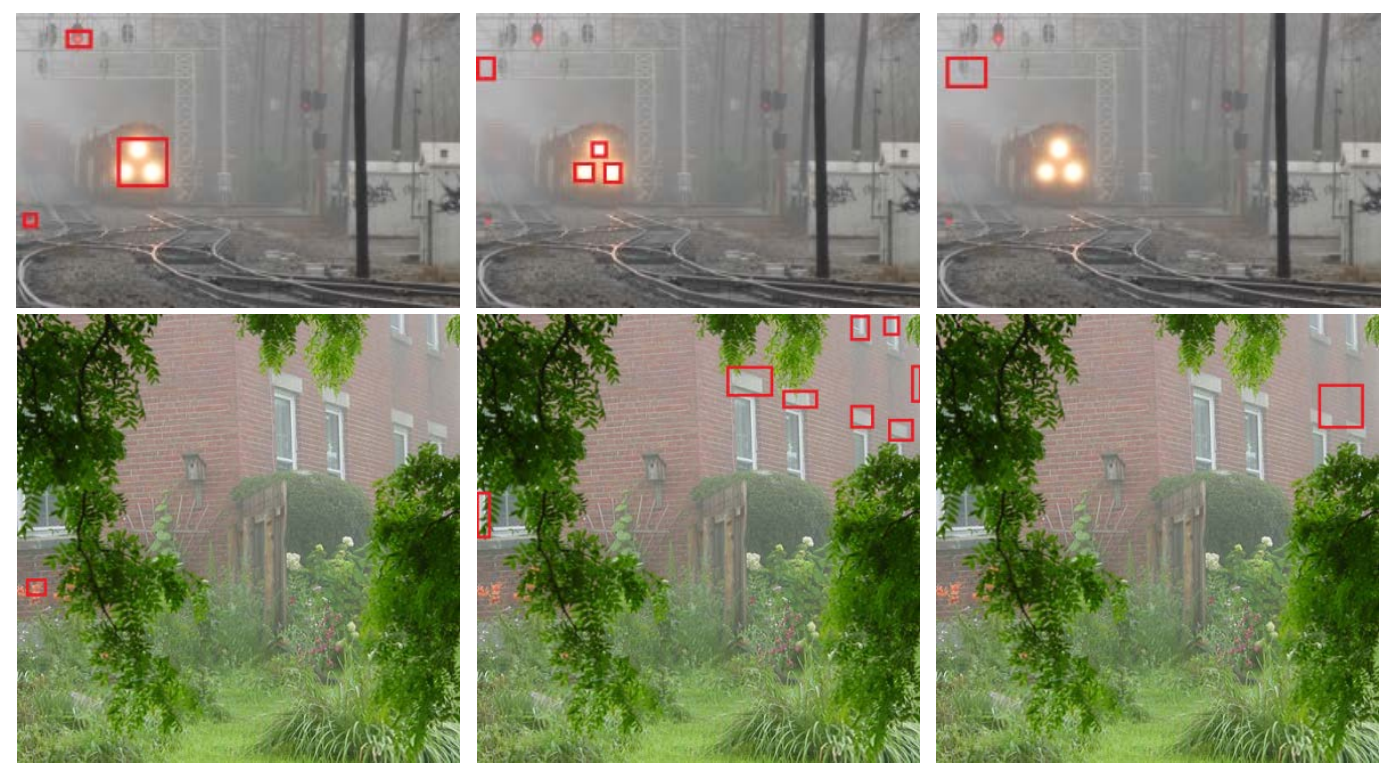

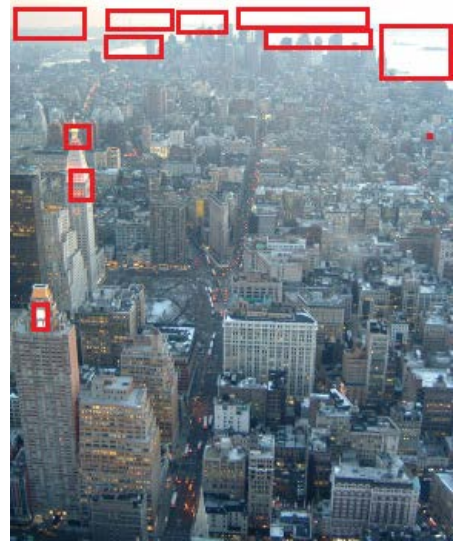

(a)

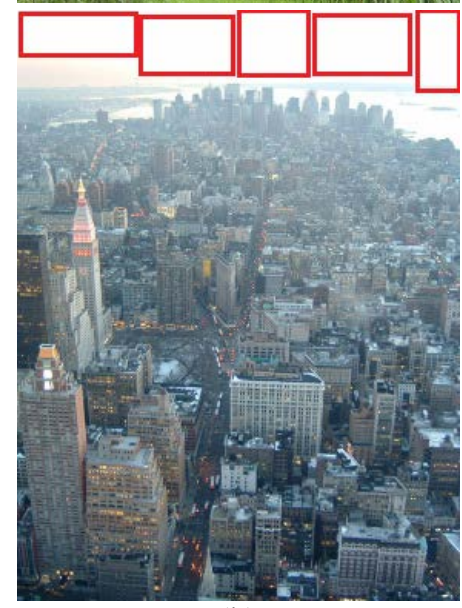

(b)

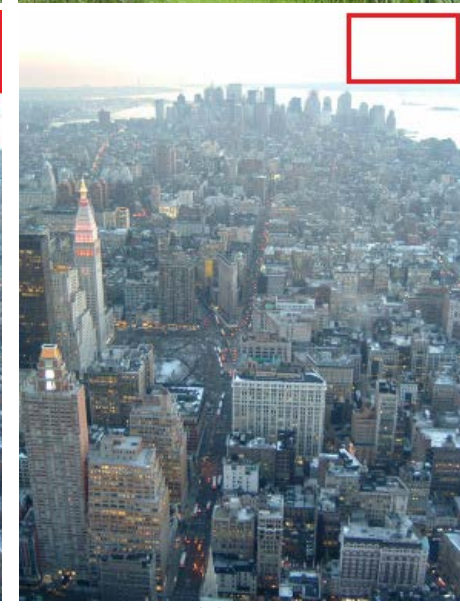

(c)

Fig. 4. Areas where the atmospheric light locates (atmospheric light estimation in different methods): (a) Tan's method, (b) He's method, and (c) quad-tree subdivision method.

Table 1. The atmospheric light values estimated by three different methods.

\begin{tabular}{|c|c|c|c|}
\hline Class & Tan's method & He's method & Quad-tree subdivision method \\
\hline \hline Train image & 1 & 1 & 0.7165 \\
\hline House image & 1 & 0.9020 & 0.7431 \\
\hline Building image & 1 & 0.9961 & 0.9216 \\
\hline
\end{tabular}

Fig. 5 gives some recovered fog-free images using He's image dehazing method with different atmospheric light values. Under comparison, we can see that larger atmospheric light value will lead to a darker recovered image. With atmospheric light $A_{c}$ estimated by quad-tree subdivision method, He's image dehazing method obtains better results. 


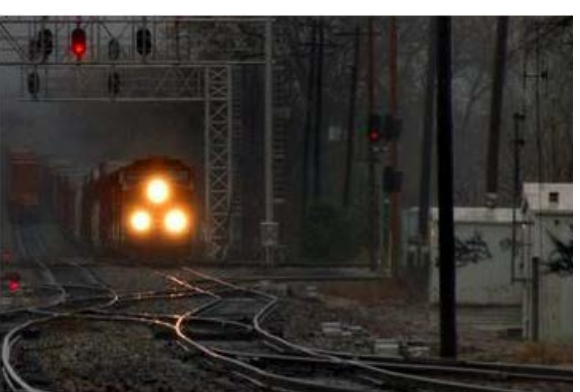

(a)

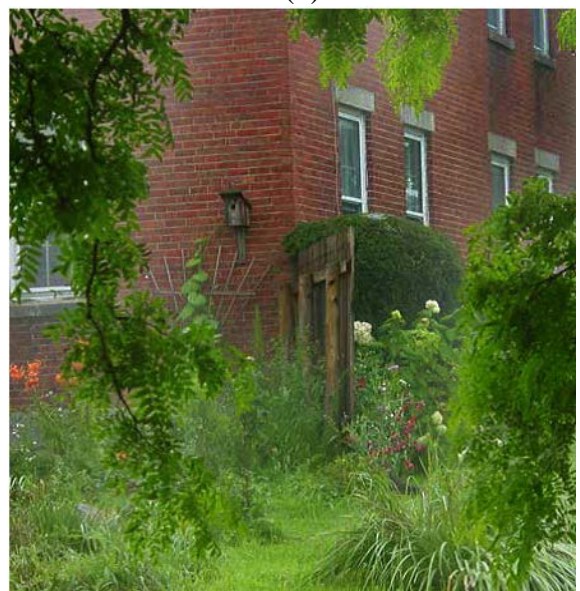

(c)

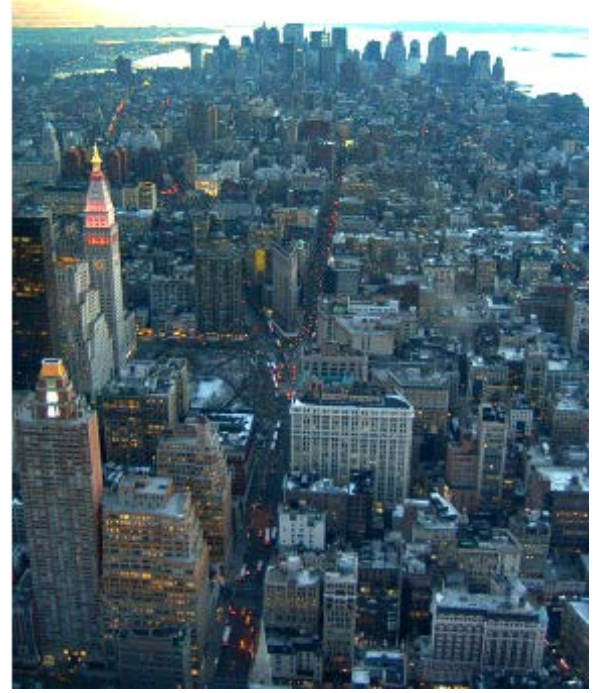

(e)

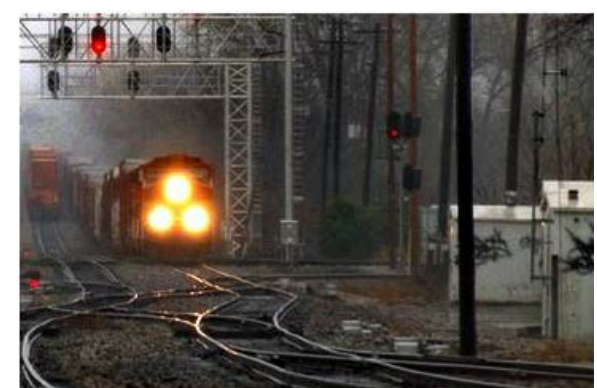

(b)

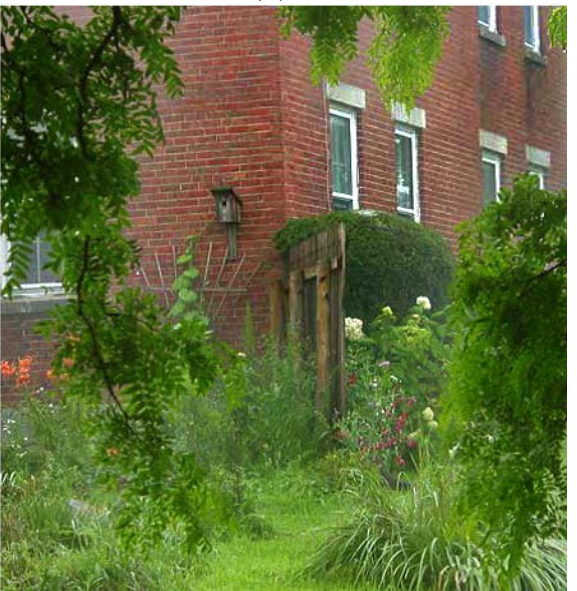

(d)

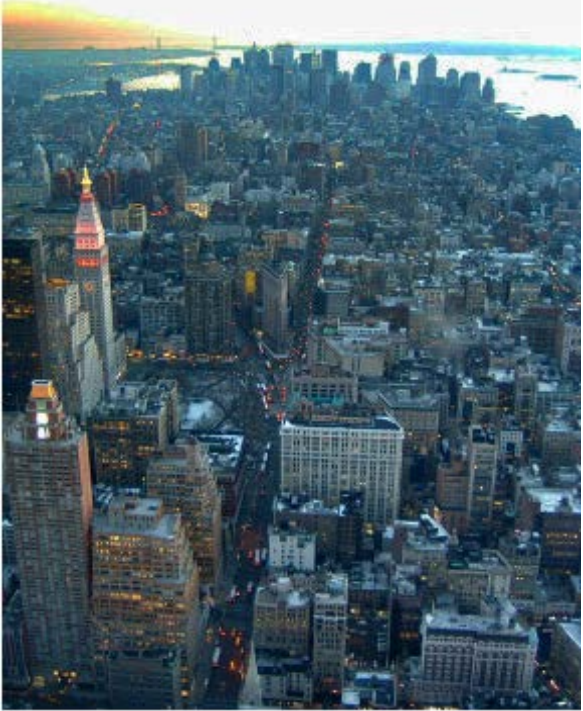

(f)

Fig. 5. Recovered fog-free images using He's image dehazing method with different atmospheric light values: From (a) to (f), the atmospheric light values are 1.0000, 0.7165, 0.9020, 0.7431, 1.0000, and 0.9216 , respectively. 


\section{Improved Image Dehazing Algorithm}

In this section, our improved image dehazing method will be described in details. Firstly, with the dark channel prior, the Eq. (14) can be rewritten as:

$$
\min _{y \in \Omega(x)}\left\{\min _{c \in\{r, g, b\}}\left[I_{c}(y)\right]\right\}=\min _{c \in\{r, g, b\}}\left\{A_{c}\right\}[1-t(x)] .
$$

Interestingly, the rewritten equation says that the dark channel of foggy image $\boldsymbol{I}$ can be considered to be the atmospheric veil. Since the intensity of the dark channel of hazy image is a rough approximation of the thickness of the haze, it will be a rough but robust estimation of the atmospheric veil. Then, on the basis of He's work and inspired by Xiao's work discussed in Section 3.1.3, we select minimum color component filtered by bilateral filter as guidance image, and use guided filter instead of guided joint bilateral filter to refine the rough atmospheric veil. The guided filter can be used as an effective edge-preserving smoothing operator but the effect is not as good as the guided joint bilateral filter. However, guided filter naturally has a fast and non-approximate linear time algorithm, regardless of the kernel size and the intensity range. Currently, it is one of the fastest edge-preserving filter. Thus, in terms of both efficiency and effect, guided filter is adopted in this paper.

In He's work, original hazy image is chosen as the guidance image for guided filtering. Since the original hazy image contains rich texture details which will be transferred into filtering output (the refined atmospheric veil or transmission map), texture details in the recovered image will be weakened. For this reason, similar to Xiao's work, the minimum color component filtered by bilateral filter is chosen to be the guidance image in our method.

Referring to the expression and derivation of [15], the left hand side of Eq. (17) can be defined as follows:

$$
\min _{y \in \Omega(x)}\left\{\min _{c \in\{r, g, b\}}\left[I_{c}(y)\right]\right\}=V_{m}(x) .
$$

In this paper, we propose a more desirable atmospheric veil $V_{m}(x)$ for image dehazing, called minimal atmospheric veil,

$$
V_{m}(x)=\min _{c \in\{r, g, b\}}\left\{A_{c}\right\}[1-t(x)]
$$

And the flow chart of our improved method is described in Fig. 6, where $A_{m}=\min _{c \in\{r, g, b\}}\left\{A_{c}\right\}$.

Fig. 7 shows the comparison between the proposed method and existed methods. The specific process of our proposed is summarized as follows.

Step 1. We first get the minimum color component map of hazy image through Eq. (6);

Step 2. A guidance image is obtained from minimum color component map under bilateral filtering; a dark channel, which can be considered to be a rough approximation of atmospheric veil, is also obtained from minimum color component map under minimum filtering;

Step 3. With rough atmospheric veil as input image, we use guided filter to transfer the structures of the guidance image to the filtering output;

Step 4. The refined atmospheric veil is acquired in Step 3. According to Eq. (3), the transmission map $t(x)$ can be obtained, which is similar to He's method. And a constant parameter $\omega(0<\omega<1)$ is introduced here to control the strength of visibility restoration. 
Step 5. Finally, substitute the estimated atmospheric light $A_{c}$ (using quad-tree subdivision method) and obtained transmission map $t(x)$ into Eq. (16) to get the fog-free image.

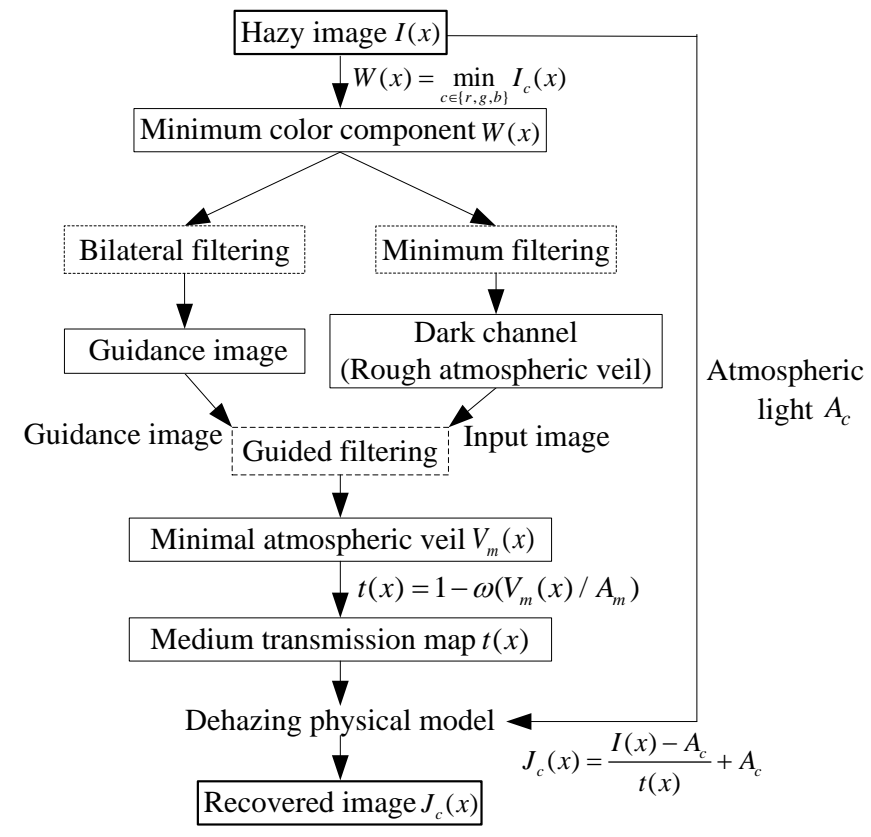

Fig. 6. The flow chart of the proposed image dehazing method.

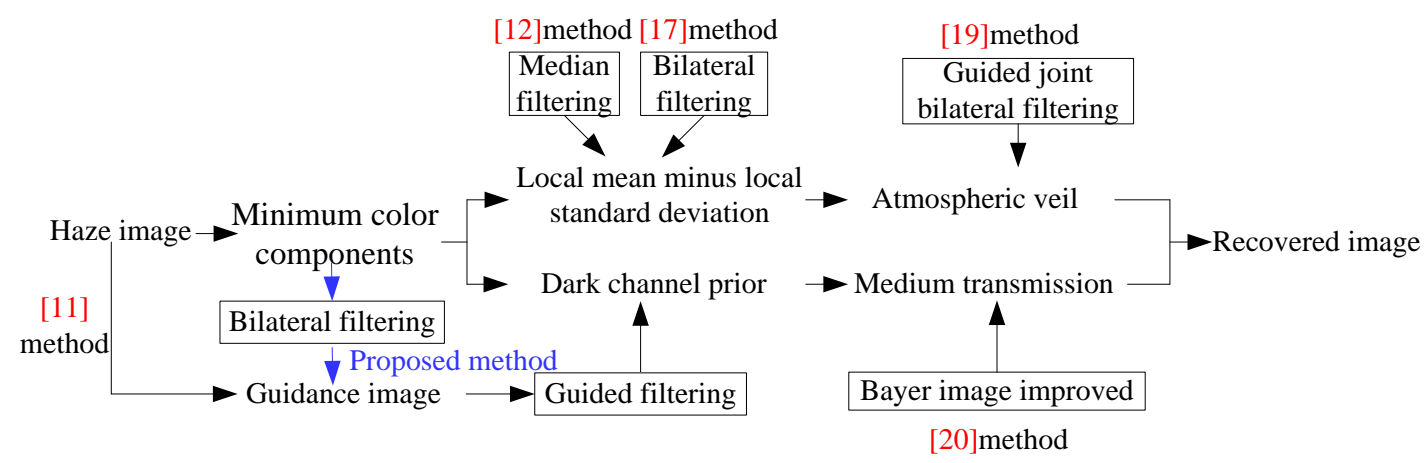

Fig. 7. The comparison between the proposed method and existed methods.

We also compare our method with He's method in Fig. 8. As we all know, in image dehazing, texture details of original foggy image are useless, which do not indicate the scene depth or the haze denseness. In view of Fig. 8(b) and Fig. 8(d), our improved method reconstructs a more desirable atmospheric veil, where texture details have been smoothed well. As a result, texture details of grassland, river bank and trunk in recovered image (Fig 8.(e)) are preserved better. 


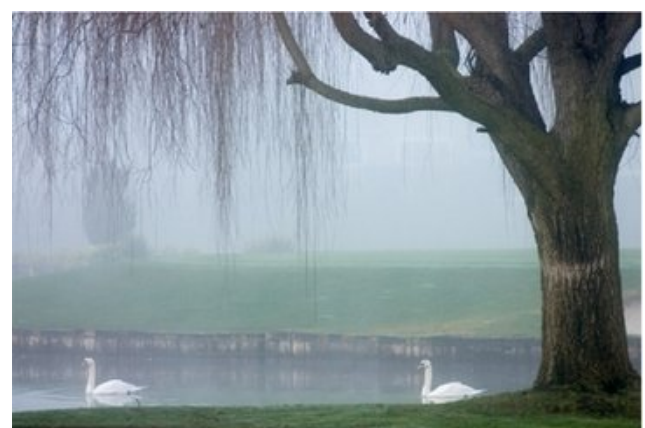

(a)

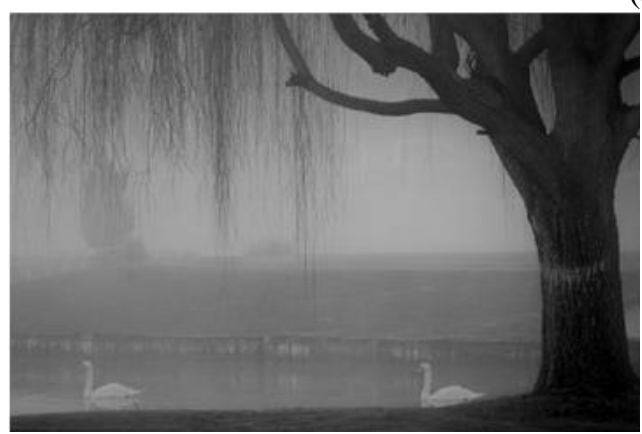

(b)

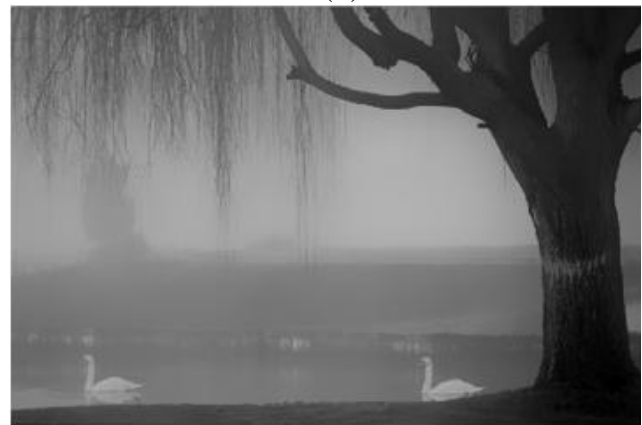

(d)

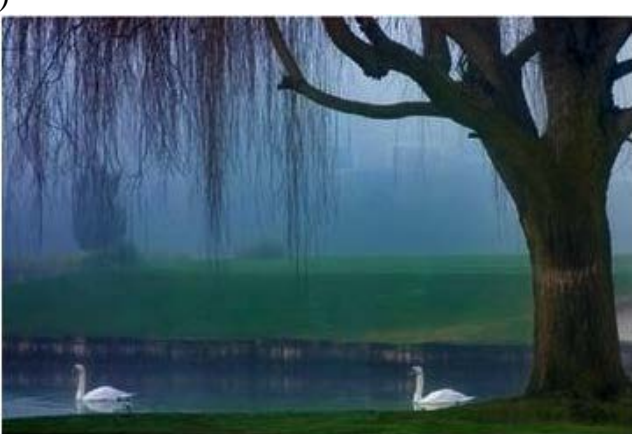

(c)

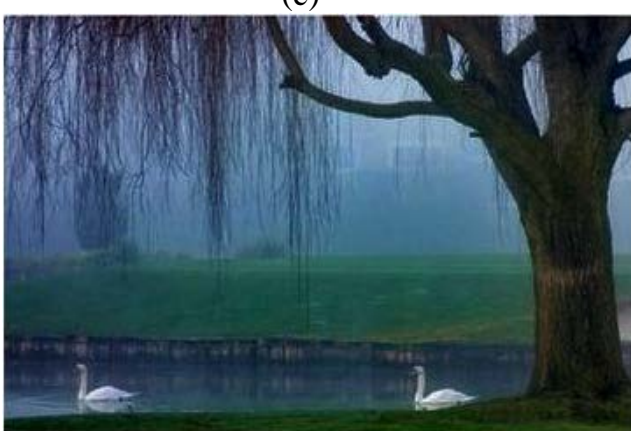

(e)

Fig. 8. The comparison between He’s method and our improved method: (a) is the input foggy image; (b) and (d) are the atmospheric veil obtained by He's method and our improved method, respectively; (c) and (e) are the dehazed images obtained by He's method and our improved method, respectively.

In practical application, through a lot of experiments, other foggy images don't conform to the principle of dark channel. When we use the method based on dark channel prior to deal with the foggy images, the color distortion would appear in the obtained images. According to the observation statistics, such images generally include the sky, the white object, the surface area and other large bright areas. Actually, even in no foggy conditions, the pixel values of these bright areas are also great. We couldn't find the dark color of these points which their pixel values are close to 0 , so the hypothesis of dark color is not established in these areas.

When the images have the bright region, atmospheric light $A_{c}$ will fall in the bright part, and we use Eq. (15) to acquire the distribution of the transmission $t$ : when $I_{c}$ is close to $A_{c}$, the values of $t$ is small. Due to the bright regions are very close to $A_{c}$, the corresponding values of $t$ will tend to be very small, but these areas are clearly not satisfied with the dark color prior, so the calculated transmission $t$ is not accurate. If we do not consider the dark 
channel prior, the derived and accurate transmission function should be Eq. (20) according to Eq. (12):

$$
t_{\text {actual }}(x)=\frac{1-\min _{y \in \Omega(x)}\left\{\min _{c \in\{r, g, b\}}\left[\frac{I_{c}(y)}{A_{c}}\right]\right\}}{1-\min _{y \in \Omega(x)}\left\{\min _{c \in\{r, g, b\}}\left[\frac{J_{c}(y)}{A_{c}}\right]\right\}} .
$$

In the bright areas, $\min _{y \in \Omega(x)}\left\{\min _{c \in\{r, g, b\}}\left[\frac{J_{c}(y)}{A_{c}}\right]\right\}$ can not be approximated to 0 , and the denominator is less than 1 . So the actual transmission is greater than transmission estimated according to the dark channel prior, that is $t_{\text {actual }}(x)>t(x)$. In order to eliminate distortion of the sky regions, we must adjust the transmission function of the bright regions, so that the estimated transmission $t(x)$ can be more consistent with the actual transmission $t_{\text {actual }}(x)$. In this paper, we use a controllable parameter $K$ to adjust transmission of each pixel. If $\left|I_{c}-A_{c}\right|<K$, the region is regard as bright regions (that is $I_{\text {sky }}(x)$ ) and transmission will be adjusted; if $\left|I_{c}-A_{c}\right|>K$, the region is regard as a region which satisfies the dark channel prior and transmission will be unchanged. The rule can be shown as follows:

$$
t_{\text {final }}(x)= \begin{cases}t(x), & \left|I_{c}-A_{c}\right|>K, \\ t_{\text {adjust }}(x), & \left|I_{c}-A_{c}\right|<K,\end{cases}
$$

where the $t_{\text {adjust }}(x)$ is the refined transmission of the bright regions, and it is defined as follows:

$$
t_{\text {adjust }}(x)=\frac{100 \times I_{\text {sky }}(x)+t(x) \times\left[255-I_{\text {sky }}(x)\right]}{255} .
$$

Fig. 9 shows the comparison between unmodified transmission and modified transmission of our improved method. From Fig. 9(b) and Fig. 9(d), we can see that if we adjust the transmission of bright regions, the transmission map is better, and the color of dehazed images looks more natural.

Compared Fig. 9(a) with Fig. 9(e), we can see that the dehazed image is a little over-saturated, so it is necessary to adjust the tone of the dehazed images to make the color and contrast of dehazed images more realistic. In this paper, we adopted method of Drago et al. [21] to conduct tone mapping. For a color channel of the dehazed images $J_{c}$, the tone mapping function can be expressed as:

$$
L J_{c}=\frac{L_{d \max } \times 0.01}{\lg \left(J_{c}^{\max }+1\right)} \times \frac{\ln \left(J_{c}+1\right)}{\ln \left\{2+\left[\left(\frac{J_{c}}{J_{c}^{\max }}\right)^{\frac{\ln (b)}{\ln (0.5)}}\right] \times 8\right\}},
$$


where $L J_{c}$ is the output of $J_{c}$ after tone mapping. $L_{d \max }$ is the the maximum brightness value that the output device can display. $J_{c}^{\max }$ is the maximum pixel value of $J_{c}$, and bias parameter $b$ is essential to adjust compression of high values and visibility of details in dark areas.

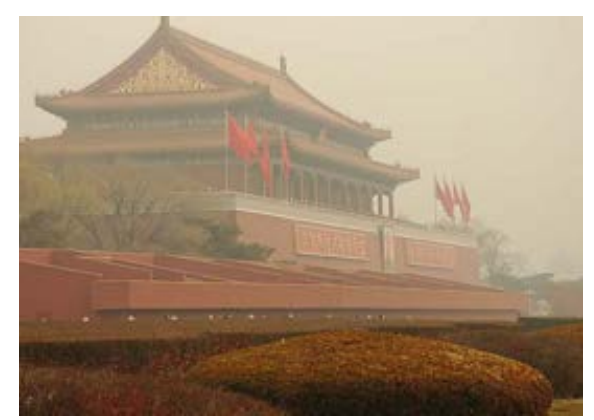

(a)

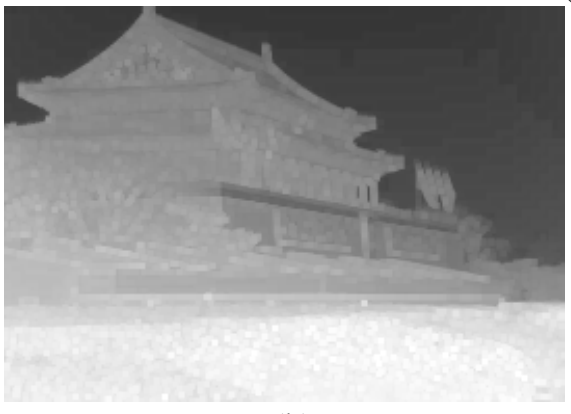

(b)

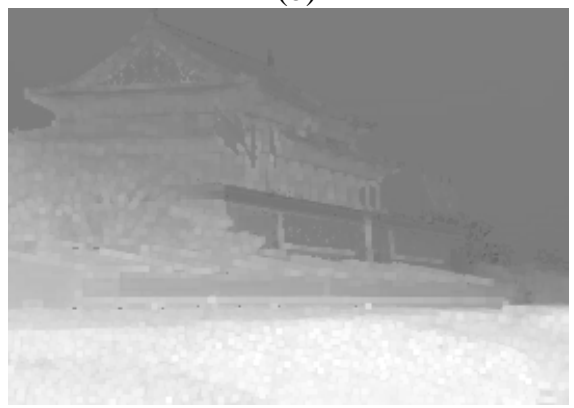

(d)

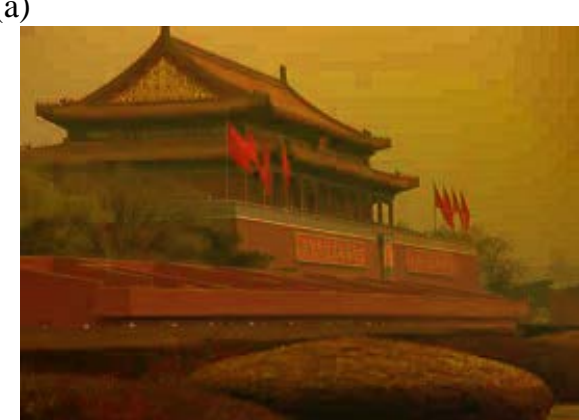

(c)

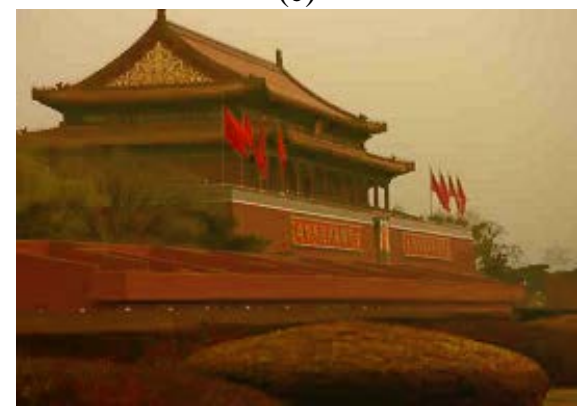

(e)

Fig. 9. The comparison between unmodified transmission and modified transmission of our improved method: (a) is the input foggy image; (b) and (d) are the transmission map obtained by unmodified transmission and modified transmission, respectively; (c) and (e) are the dehazed images obtained by unmodified transmission method and modified transmission method, respectively.

Fig. 10 shows the contrast effect figures before and after tone mapping. After the tone mapping, the phenomenon of over-saturated can be eased. 


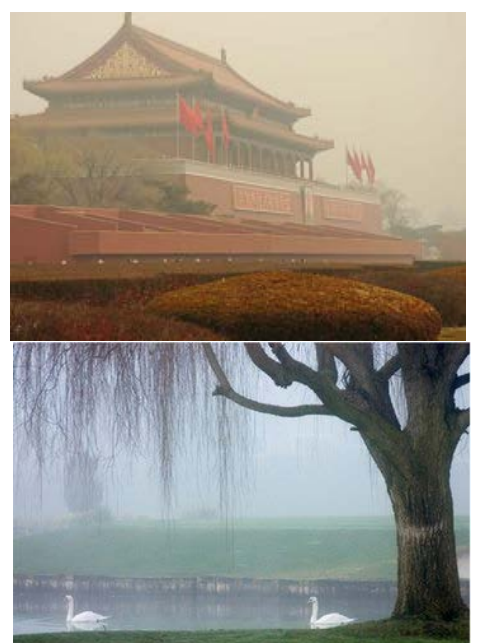

(a)

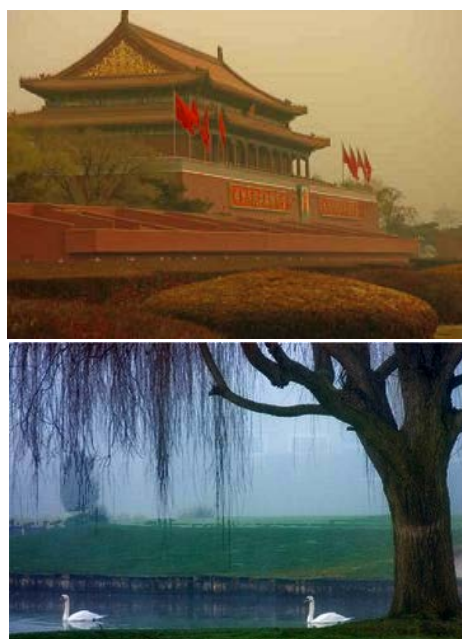

(b)

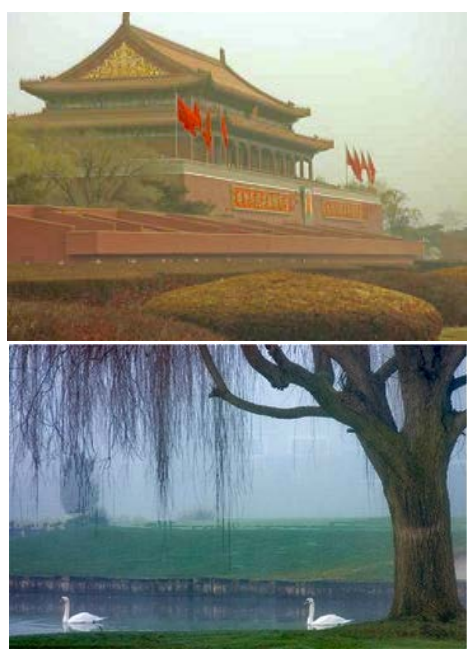

(c)

Fig. 10. Comparison between before and after tone mapping: (a) Original foggy images, (b) Recovered images before tone mapping, and (c) Recovered images after tone mapping.

\section{Experimental Results}

In this section, we show and discuss the results of the proposed method, and compared with several state of the art image dehazing methods in the image restoration quality. Throughout this paper, all experiments are conducted with MATLAB 2010a. Fig. 11 gives some simulation results of single image dehazing corresponding to Tarel's method, He's method and our improved method. It can be seen that our proposed algorithm has achieved satisfactory dehazing effect: the brightness of recovered images looks more natural; edges and texture details in dehazed image are kept better, especially in the close-range area and many hidden details in prospective areas are also visible in the restored images; there is no significant color error in the white objects and sky regions. Moreover, compared to the Tarel's results, halo artifacts are also significantly small in our results.
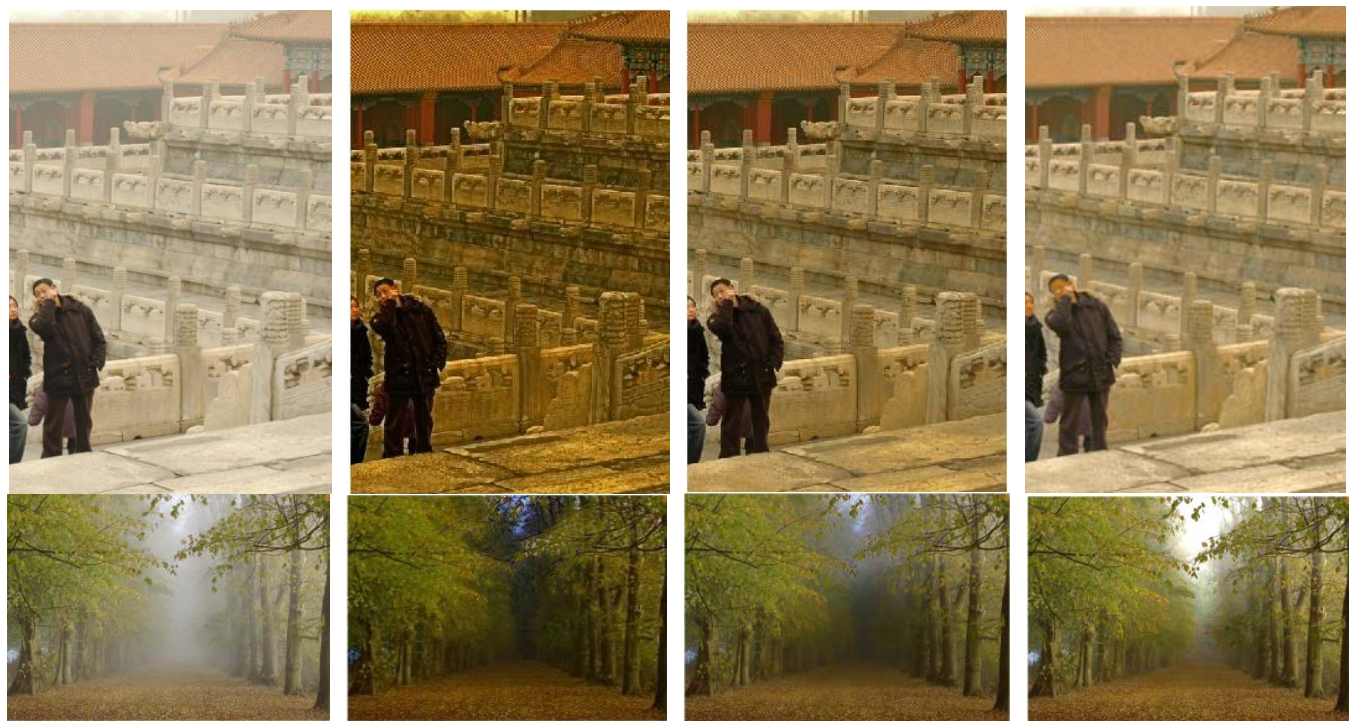


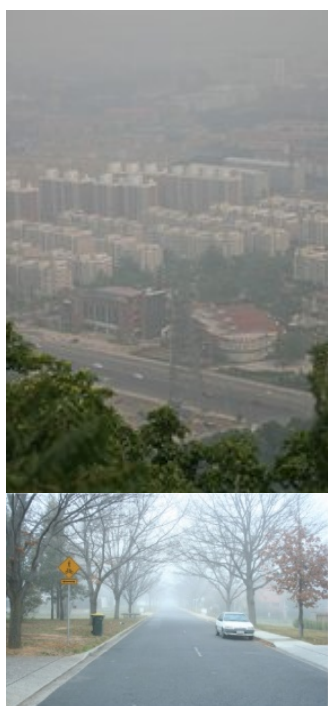

(a)

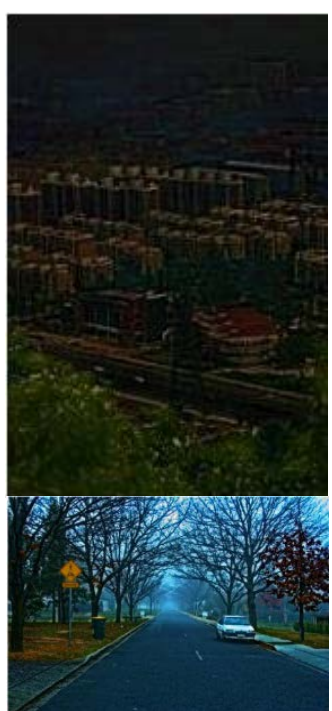

(b)

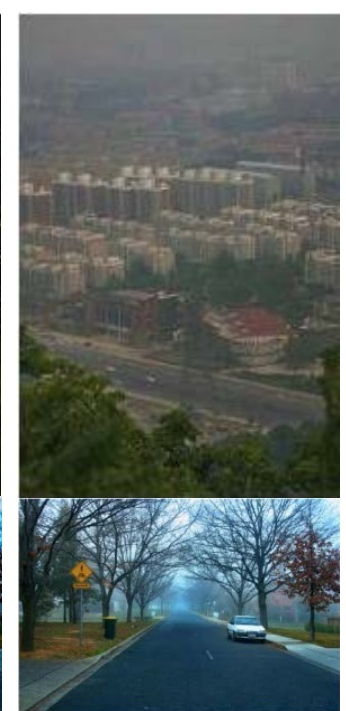

(c)

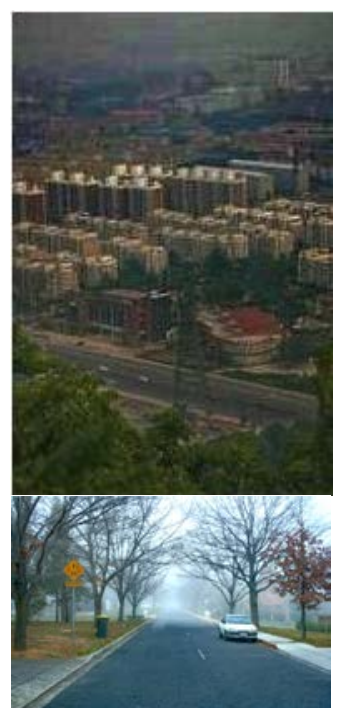

(d)

Fig. 11. Comparison with other methods: (a) is the original foggy images; (b), (c), and (d) are recovered images using Tarel's method, He’s method, and proposed method, respectively.

\section{Conclusion}

We can conclude that on the basis of atmospheric scattering model, the key to dehaze a foggy image is that how to accurately estimate the atmospheric light and atmospheric veil (or transmission map) from a single hazy image. With an in-depth comparative study on previous fog removal schemes, our proposed method in this paper is a beneficial exploration to further improve the quality of the recovered image through estimating the atmospheric light and atmospheric veil more accurately. Besiedes, we also adjust the transmission of the sky regions and conduct the tone mapping for the obtained images to improve the their quality further. Under the comparison of our results with other state of the art image dehazing methods, our results are more satisfactory.

As the bilateral filter, the guided filter and the minimum filter can be implemented parallelly, thus, our method can be further accelerated using GPU (Graphics Processing Unit). To be sure, in the further work, we will continue to devote ourselves to seeking a more effective and efficient method to restore the images which suffer from the fog. On the other hand, we will research the relationship between image dehazeing methods and image coding to obtain one better image dehazing method for images transmission of surveillance systems.

\section{Acknowledgments}

This work was supported by the National Natural Science Foundation of China (Grant No. 61201371), the Natural Science Foundation of Shandong Province, China (Grant No. ZR2015PF004), the promotive research fund for excellent young and middle-aged scientists of Shandong Province, China (Grant No. BS2013DX022), the Fundamental Research Funds of Shandong University (Grant No. 2014ZQXM008), and the Development Program for Outstanding Young Teachers in School of Mechanical, Electrical and Information Engineering, Shandong University, Weihai. The authors thank Fanfan Yang, Heng Zhang, and Yunpeng Zhang for their kind help and valuable suggestions. The authors also thank the 
anonymous reviewers and the editor for their valuable comments to improve the presentation of the paper.

\section{References}

[1] P. Babu and V. Rajamani, "Contrast enhancement using real coded genetic algorithm based modified histogram equalization for gray scale images," International Journal of Imaging Systems and Technology, vol. 25, no. 1, pp. 24-32, Mar. 2015. Article (CrossRef Link).

[2] B. Xie, F. Guo, and Z. X. Cai, "Improved single image dehazing using dark channel prior and multi-scale Retinex," in Proc. of the International Conference on Intelligent System Design and Engineering Application, Changsha, China, Oct. 13-14, vol. 1, pp. 848-851, 2010. Article (CrossRef Link).

[3] S. G. Narasimhan and S. K. Nayar, "Contrast restoration of weather degraded images," IEEE Transactions on Pattern Analysis and Machine Intelligence, vol. 25, no. 6, pp. 713-724, Jun. 2003. Article (CrossRef Link).

[4] D. Miyazaki, D. Akiyama, M. Baba, R. Furukawa, S. Hiura, and N. Asada, "Polarization-based dehazing using two reference objects," in Proc. of the IEEE International Conference on Computer Vision Workshops, Sydney, Australia, Dec. 2-8, pp. 852-859, 2013. Article (CrossRef Link).

[5] S. G. Narasimhan and S. K. Nayar, "Contrast restoration of weather degraded images," in Proc. of the ACM SIGGRAPH Asia Courses, Singapore, Singapore, Dec. 10-13, 12 pages, Article number: 70, 2008. Article (CrossRef Link).

[6] J. Kopf, B. Neubert, B. Chen, M. Cohen, D. Cohen-Or, O. Deussen, M. Uyttendaele, and D. Lischinski, "Deep photo: Model-based photograph enhancement and viewing," ACM Transactions on Graphics, vol. 27, no. 5, 10 pages, Article number: 116, Dec. 2008. Article (CrossRef Link).

[7] R. T. Tan, "Visibility in bad weather from a single image," in Proc. of the IEEE Conference on Computer Vision and Pattern Recognition, Anchorage, USA, Jun. 23-28, 8 pages, Article number: 4587643, 2008. Article (CrossRef Link).

[8] R. Fattal, "Single image dehazing," ACM Transactions on Graphics, vol. 27, no. 3, 9 pages, Article number: 72, Aug. 2008. Article (CrossRef Link).

[9] K. M. He, J. Sun, and X. O. Tang, "Single image haze removal using dark channel prior," in Proc. of the IEEE Computer Society Conference on Computer Vision and Pattern Recognition Workshops, Miami, USA, Jun. 20-25, pp. 1956-1963, 2009. Article (CrossRef Link).

[10] A. Levin, D. Lischinski, and Y. Weiss, "A closed-form solution to natural image matting," IEEE Transactions on Pattern Analysis and Machine Intelligence, vol. 30, no. 2, pp. 228-242, Feb. 2008. Article (CrossRef Link).

[11] K. M. He, J. Sun, and X. O. Tang, "Guided image filtering," IEEE Transactions on Pattern Analysis and Machine Intelligence, vol. 35, no. 6, pp. 1397-1409, Jun. 2013. Article (CrossRef Link).

[12] J. P. Tarel and N. Hautiere, "Fast visibility restoration from a single color or gray level image," in Proc. of the IEEE 12th International Conference on Computer Vision, Kyoto, Japan, Sep. 29-Oct. 2, pp. 2201-2208, 2009. Article (CrossRef Link).

[13] G. Y. Wang, G. H. Ren, L. H. Jiang, and T. F. Quan, "Single image dehazing algorithm based on sky region segmentation," Information Technology Journal, vol. 12, no. 6, pp. 1168-1175, Jun. 2013. Article (CrossRef Link).

[14] Z. G. Li, J. H. Zheng, Z. J. Zhu, W. Yao, and S. Q. Wu, "Weighted guided image filtering," IEEE Transactions on Image Processing, vol. 24, no. 1, pp. 120-129, Jan. 2015. Article (CrossRef Link).

[15] Z. G. Li and J. H. Zheng, "Edge-preserving decomposition-based single image haze removal," IEEE Transactions on Image Processing, vol. 24, no. 12, pp. 5432-5441, Dec. 2015. Article (CrossRef Link). 
[16] Q. S. Zhu, J. M. Mai, and L. Shao, "A fast single image haze removal algorithm using color attenuation prior," IEEE Transactions on Image Processing, vol. 24, no. 11, pp. 3522-3533, Nov. 2015. Article (CrossRef Link).

[17] K. Sun, B. Wang, Z. Q. Zhou, and Z. H. Zheng, "Real time image haze removal using bilateral filter,” Transactions of Beijing Institute of Technology, vol. 31, no. 7, pp. 810-813, 822, Jul. 2011. (in Chinese) Article (CrossRef Link).

[18] C. Tomasi and R. Manduchi, "Bilateral filtering for gray and color images," in Proc. of the IEEE Conference on Computer Vision, Bombay, India, Jan. 4-7, pp. 839-846, 1998. Article (CrossRef Link).

[19] C. X. Xiao and J. J. Gan, "Fast image dehazing using guided joint bilateral filter," Visual Computer, vol. 28, no. 6-8, pp. 713-721, Jun. 2012. Article (CrossRef Link).

[20] J. T. Lou, W. Wang, Y. L. Li, and M. J. Zhang, "A fast approach to remove the haze from a single Bayer image," Journal of National University of Defense Technology, vol. 35, no. 6, pp. 109-115, Dec. 2013. Article (CrossRef Link).

[21] F. Drago, K. Myszkowski, T. Annen, and N. Chiba, "Adaptive logarithmic mapping for displaying high contrast scenes,” Computer Graphics Forum, vol. 22, no. 3, pp. 419-426, Sep. 2003. Article (CrossRef Link). 

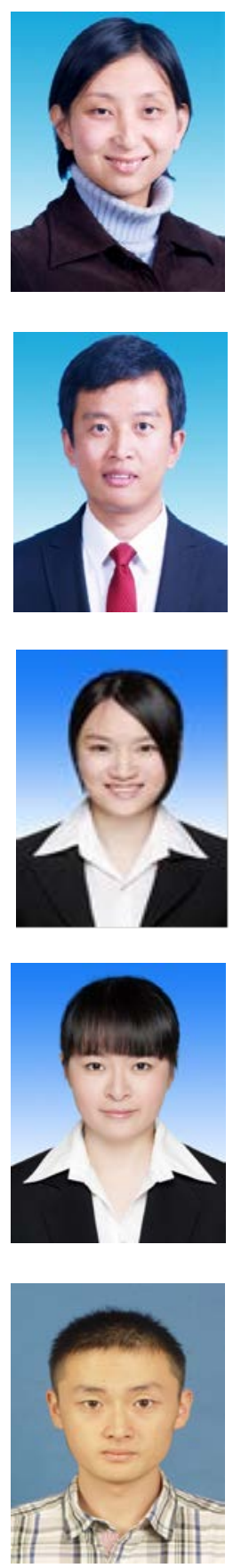

Xiao Zhou received her B.E. degree in automation from Nanjing University of Posts and Telecommunications, China in 2003, her M.E. degree in information and communication engineering from Inha University, Korea in 2005, and her Ph.D. degree in information and communication engineering from Tsinghua University, China in 2013. Now she is a lecturer in the School of Mechanical, Electrical and Information Engineering, Shandong University, Weihai, China. Her current research interests include wireless communication technology, digital image processing and analysis.

Chengyou Wang received his B.E. degree in electronic information science and technology from Yantai University, China in 2004, and his M.E. and Ph.D. degree in signal and information processing from Tianjin University, China in 2007 and 2010 respectively. Now he is an associate professor and supervisor of postgraduate in the School of Mechanical, Electrical and Information Engineering, Shandong University, Weihai, China. His current research interests include digital image/video processing and analysis, pattern recognition and machine learning.

Liping Wang received her B.E. degree in communication engineering from Shandong University, Weihai, China, in 2014. She is currently pursuing her M.E. degree in signal and information processing at Shandong University, China. Her current research interests include digital image processing and analysis techniques.

Nan Wang received her B.S. degree in electronic information science and technology from Shandong University, Weihai, China in 2015. She is currently pursuing her M.E. degree in information and communication engineering at Tianjin University, China. Her current research interests include digital image processing and analysis techniques.

Qiming Fu received his B.S. degree in electronic information science and technology from Shandong University, Weihai, China in 2013. He is currently pursuing his M.E. degree in electronics and communication engineering at Shandong University, China. His current research interests include digital image processing and analysis techniques. 